\title{
A look into the nature and causes of human errors in the intensive care unit*
}

\author{
Y Donchin, D Gopher, M Olin, Y Badihi, M Biesky, C L Sprung, R Pizov, S Cotev
}

Qual Saf Health Care 2003;12:143-148

\begin{abstract}
Objectives: The purpose of this study was to investigate the nature and causes of human errors in the intensive care unit (ICU), adopting approaches proposed by human factors engineering. The basic assumption was that errors occur and follow a pattern that can be uncovered.

Design: Concurrent incident study.

Setting: Medical-surgical ICU of a university hospital.

Measurements and main results: Two types of data were collected: errors reported by physicians and nurses immediately after an error discovery; and activity profiles based on 24-h records taken by observers with human engineering experience on a sample of patients. During the 4 months of data collection, a total of 554 human errors were reported by the medical staff. Errors were rated for severity and classified according to the body system and type of medical activity involved. There was an average of 178 activities per patient per day and an estimated number of 1.7 errors per patient per day. For the ICU as a whole, a severe or potentially detrimental error occurred on average twice a day. Physicians and nurses were about equal contributors to the number of errors, although nurses had many more activities per day.

Conclusions: A significant number of dangerous human errors occur in the ICU. Many of these errors could be attributed to problems of communication between the physicians and nurses. Applying human factor engineering concepts to the study of the weak points of a specific ICU may help to reduce the number of errors. Errors should not be considered as an incurable disease, but rather as preventable phenomena.
\end{abstract}

See end of article for authors' affiliations

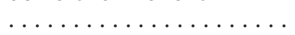

rar nvestigations of the nature of human errors in hospitals are rare. It is human nature not to report errors, and the medical profession is no different from other professions. The importance of error prevention was recognized by anesthesiologists with the publication of error analysis and critical incidences in the operating theater. ${ }^{1}$ (Anesthesiology is one of the few fields to publish such a study.) The paucity of published investigation in this area may be related, at least in part, to the fear of legal liability.

Great efforts have been invested in the industrial sector in the analysis of job requirements and the design of workplaces, equipment, and the physical environment for the benefit of workers. In air traffic control, for instance, human factors have been studied extensively. ${ }^{2}$ In contrast, almost no attention has been given to human factor considerations in the hospital setting. As malpractice premiums have increased, hospitals tend to spend more time preparing themselves against liability claims rather than actively trying to avoid errors. A recent review ${ }^{3}$ concluded that "reducing the incidence of the events will require identification of their causes and developing methods to prevent errors or reduce their effect".

Observations during routine daily activities in the intensive care unit (ICU) demonstrated that mistakes do occur, sometimes with severe consequences. ${ }^{45}$ The present prospective study investigated the nature and causes of human errors in an active ICU in an effort to develop a methodology to avoid or reduce error frequency and impact. We report two phases of a concurrent incident study describing the activity profile of the ICU and analysis of the human errors collected within the framework of these activities.

*This is a reprint of a paper that appeared in Critical Care Medicine, 1995, Volume 23, pages 294-300.

\section{MATERIALS AND METHODS}

The Hadassah-Hebrew University Medical Center at EinKerem, Jerusalem is a tertiary-care teaching hospital of 650 beds. It has six critical care units (pediatric, neurosurgical, postcardiac surgical, coronary care, burn, and medicalsurgical). The medical-surgical ICU is a six-bed unit, with additional "overflow" beds available in the nearby recovery room. The yearly occupancy rate of this ICU reaches $110 \%$. The medical-surgical ICU is staffed by a director, a senior attending physician, and rotating residents from the departments of anesthesia, surgery, and occasionally from internal medicine. The patient/nurse ratio for all shifts is $2: 1$, regardless of the severity or the number of patients.

The ICU mortality rate for the period in which this study was conducted was $12 \%$, a rate that has not varied significantly in the last 10 years.

Monitor-derived data and other measurements (i.e. fluid intake and urine output, response of pupils to light, Glasgow coma score) were recorded on a flow sheet. On the same flow sheet, physicians and nurses recorded additional events, such as emergency endotracheal intubation, vascular cannulation, or cardiopulmonary resuscitation. Important laboratory results and blood gas analyses were recorded as well. Nursing rounds were made three times daily at the patient's bedside on the occasion of the change of each shift. Physician work rounds were carried out by attending and resident staff in the morning; the rounds were performed again, in the late afternoon, by the on-duty physician for that night.

Daily physician's orders were written during the morning rounds, after examining and assessing the patient's status over the last 24 hours. Orders may be modified at any time, as dictated by changes in the patient's status.

For the purpose of the study, a human error was defined as a deviation from standard conduct, as well as addition or 
omission of actions relating to standard operational instructions or routines of the unit. Examples of human error included failure to observe inappropriate rates of flow of intravenous fluids, administration of an erroneous drug or drug dosage, order to administer a drug intramuscularly rather than intravenously, errors in calculating drug administration flow rates, and failure to properly perform a written order. Medical decisions were not addressed. Therefore, retrospectively inappropriate decisions were not considered errors. The observations and data collection took place over 4 months in 1989. Two types of data were collected: error reports and activity profiles.

\section{Error reports}

Errors were reported by physicians and nurses immediately as they were discovered. These errors were recorded on a form that was produced for the purpose of this study. The form did not become part of the patient's medical record. Before data collection had begun, several meetings were held between unit staff and members of the research team. During these meetings, the objectives of the study were discussed and the major elements of the error recording form were determined. The attitude of the staff toward the study was highly positive, and their full cooperation was assured.

The error report form included time of occurrence, time of discovery, sectional identity (e.g. physician, nurse, or other) of the person who committed the error and the sectional identity of the person who discovered it, a short description of the error and its presumed cause, and the number of invasive catheters and mode of ventilation. Errors were further evaluated during data analysis as to their nature and severity.

Each discovered error was rated independently by three senior medical personnel-two physicians (YD and RP) and one ICU nurse (MB) —on a 5-point severity scale, where 1 was least serious and 5 most serious. A severity error of 5 was an error that if not discovered could have caused severe injury and subsequent deterioration in clinical status. An example of a severe error is error 292. A blood sample was sent for typing and cross-matching to the blood bank with the wrong patient label on the test tube. This error was discovered immediately at the blood bank and no harm was done; however, had it not been discovered in time, the error might have been fatal. Another example of a severe error is error 453. A nurse received an order to prepare $50 \mathrm{mg}$ of ephedrine for intravenous administration. Only because the order was delayed did the physician notice that the nurse was trying to open 50 ampules of $1 \mathrm{mg}$ of epinephrine! Severity level 1 or 2 was assigned in cases where calculation of urine output or the total fluid intake for 24 hours was wrong.

\section{Activity profile}

Twenty-four hour continuous bedside observations were conducted on a randomly selected group of 46 patients who were representative of the patient population in the unit. The observations were performed by investigators from the Technion who are not medically trained. They received training for the project from a senior ICU nurse (MB) who also supervised their activity. The same nurse took part in the ongoing observations but was not a part of the ICU nursing staff during the observation period. Activity was defined as any interaction involving the patient and his or her immediate bedside surroundings, and all activities around the patient's bed during the 24-hour observations were recorded. Examples of recorded activities include replacing intravenous fluids, calibrating a transducer, or administering a drug. Each single interaction, regardless of the time required, was counted as a single interaction. The investigators also recorded any human errors they detected in the course of the observations.

Activities were recorded on a form prepared for the study as a result of working with the senior ICU nurse on two pilot cases. Each activity was recorded, along with its time, type, and nature, as well as the member of the staff performing the activity. These observations provided an essential baseline profile of daily activity in the ICU, as well as a reference point for the rate of errors performed. They also served as an independent validation of the accuracy and completeness of the medical and nursing staff records of human errors.

Activities were divided into three categories, according to the following criteria. Planned activities included the performance of routine standing orders. Initiated activities included additional treatments and procedures that were not an integral part of the routine. Reactive activities included activities in direct response to changes in the patients' clinical status.

A special coding system was developed to encode the 24-hour records and the error reports. The resultant database was submitted to statistical analysis.

\section{Statistical methods}

The main data collection period extended over 4 months in 1989. Frequency distributions, average activity, error rates, and percentages were computed and cross-tabulated using statistical software (SAS, Cary, NC). The same software was used in all statistical analyses. This analysis was employed to test the statistical significance of differences between frequencies of events in categories. Pearson product-moment correlation coefficients were computed to assess the degree to which the judgment of experts agreed regarding the severity of the errors. Comparisons between the average number of errors per hour at different times of the day were conducted using $t$ tests in a planned comparison model.

\section{RESULTS}

\section{Activity profile of the intensive care unit}

The Technion observers recorded a total of 8178 activities during their 24-hour surveillances of 46 patients (an average of 178 activities per patient per day). Seventy eight errors were recorded during these observations $(0.95 \%$ of activities). Activities performed by staff members had the following distribution: $382(4.7 \%)$ activities were performed by a single physician, and 174 (2.2\%) activities involved two or more physicians; 6857 (84\%) activities were performed by a single nurse, and two nurses together accounted for $228(2.7 \%)$ activities; nurses and physicians together were involved in 256 (3\%) activities; technicians, other paramedical personnel, and visiting family members contributed $281(3 \%)$ of the total number of activities.

Of the 382 activities performed by physicians, 181 (47\%) were classified as planned, 71 (19\%) were classified as initiated, and 126 (33\%) were classified as reactive. (Four activities could not be classified.) Of the 6857 activities performed by nurses, 6316 (92\%) were planned, with only $361(5.5 \%)$ initiated, and $164(2.5 \%)$ reactive. Figure 1 describes the diurnal distribution of the activities of physicians and nurses throughout the 24 hours. In groups, peak activity occurs during late morning and early afternoon hours (10.00 to 13.00 hours). However, while the number of activities performed by physicians decreased sharply outside of this "window," nurses maintained a high rate of activity at all hours. Methods used to record all of the patients' daily activities included the patient's bedside flow sheet only (47\%), the physician's order sheet only $(7 \%)$, and both forms $(28 \%)$. Eighteen percent of the activities were not recorded in any form.

On the last 15 of the 4624 -hour observations, special attention was devoted to verbal communication among staff members. Of 3018 activities recorded for these 15 patients, verbal communication was observed only in 291 (9\%) activities. Most communications were exclusively among physicians or exclusively among nurses. Only in $60(2 \%)$ of the recorded activities did physicians communicate verbally with nurses. 


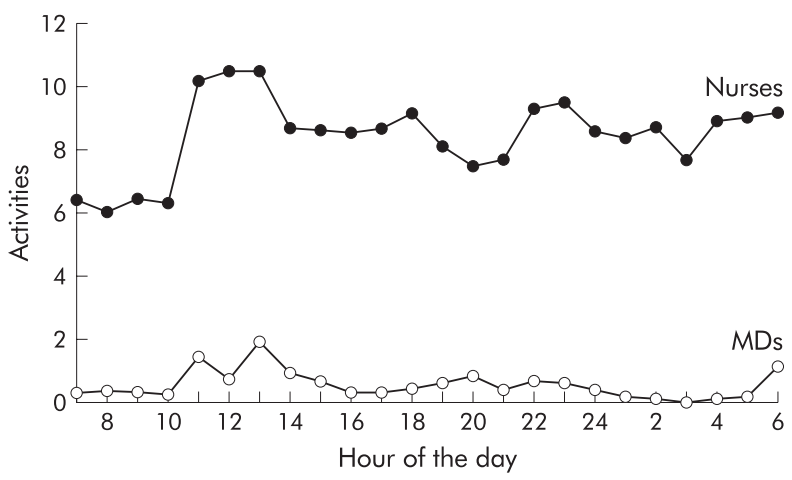

Figure 1 Diurnal distribution of physician and nurse activities in the intensive care unit. Activity was measured as activity per patient per hour. Peak activity occurred during the late morning. While the intensity of activity performed by physicians decreased sharply, nurses maintained a high rate of activity.

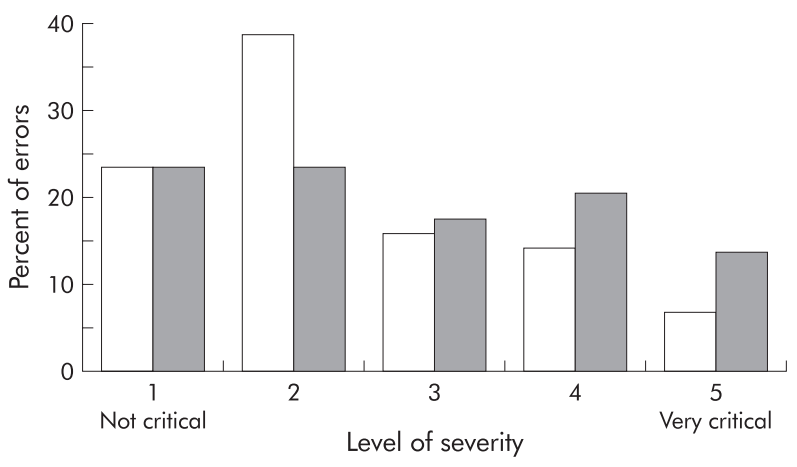

Figure 2 Distribution of the severity of errors. The graph displays the joint distribution and the separate rating of errors performed by physicians (open bars) and nurses (shaded bars).

\section{Analysis of errors}

During the 4 months of data collection, 554 human errors were recorded. Physicians and nurses recorded 476 errors on the special form that they discovered during their routine work. Seventy eight additional errors were detected by the Technion observers during their observations. Forty eight of the 78 errors were also reported by the medical staff. All statistical analysis was adjusted for this overlap.

The severity rating of errors showed that 147 (29\%) errors were in categories 4 and 5-that is, errors that could potentially cause significant deterioration in patient's status or even death. The correlation coefficients between the severity ratings of the three raters (YD, RP, and $\mathrm{MB}$ ) were 0.63 to 0.70 . Although correlation coefficients at this level imply only a moderate degree of agreement between raters, a closer examination of the differences between judgments showed that the judgments differed by more than l severity score point in only $3 \%$ of ratings. Figure 2 depicts the separate and joint rating of errors by physicians and nurses. Of the total number of errors reported by the ICU medical staff, 206 (46\%) errors were committed by physicians and 240 (54\%) errors were committed by nurses. This distribution was the same whether the reporters of the errors were physicians or nurses. A similar distribution for the two classes of staff members was also found when the 78 errors detected by the Technion observers were analyzednamely, 32 (40\%) errors were committed by physicians and 46 $(60 \%)$ errors were committed by nurses.

Diurnal distribution of errors over the hours of the day is plotted in fig 3. On average, more errors per hour were committed during the day than during the night. Of the total number of errors committed by physicians, $72.7 \%$ occurred in the day and only $21.3 \%$ occurred at night $(\mathrm{p}<0.027)$. Of the

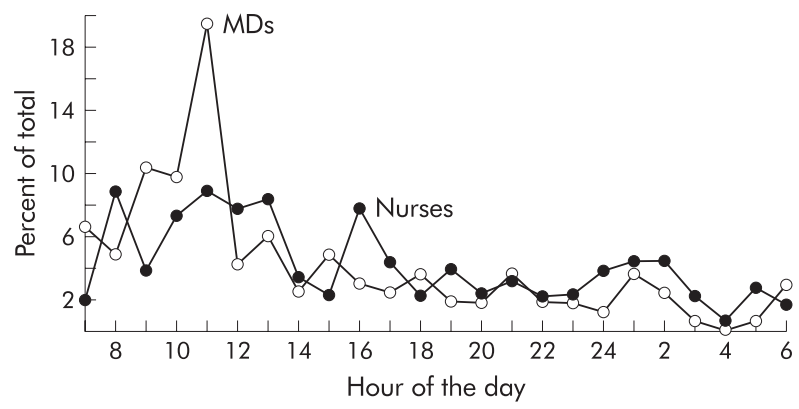

Figure 3 Diurnal distribution of commitment of errors. The errors are expressed as percent of the total errors.

Table 1 Error rate according to the activity ratio

\begin{tabular}{llllll}
\hline & Activity & $\%$ & Errors & $\%$ & Ratio $^{a}$ \\
\hline Nervous system & 202 & 2.9 & 22 & 5.1 & 1.76 \\
Breathing & 1783 & 25.9 & 98 & 22.8 & 0.88 \\
Cardiovascular & 1291 & 18.7 & 23 & 5.5 & 0.29 \\
Digestive & 1002 & 14.6 & 10 & 2.3 & 0.16 \\
Urine & 700 & 10.2 & 10 & 2.3 & 0.22 \\
Input & 1644 & 24.1 & 246 & 57.3 & 2.38 \\
Output & 244 & 3.6 & 20 & 4.7 & 1.31 \\
Total & 6892 & 100 & 429 & 100 & - \\
\hline \multicolumn{7}{l}{ The ratio is percent of all errors divided by percent of all activities. } \\
\hline \multicolumn{7}{l}{}
\end{tabular}

total number of errors committed by nurses, $68 \%$ occurred during the day and $32 \%$ occurred during the night $(\mathrm{p}<0.002)$. Errors that were attributed to physicians had one distinct peak, generally corresponding to the time window of their peak activity, between 10.00 and 12.00 hours. Errors attributed to nurses had a similar morning peak, with a 1-hour lag after the physicians' rounds. In addition, nurses had three secondary peaks at $07.30,15.30$, and 24.00 hours, corresponding to the times of the nurses' shift change.

Verbal communications between physicians and nurses were recorded in 205 (37\%) of the error reports. This percentage is surprisingly high when contrasted with the finding that verbal communications between physicians and nurses were observed only in $2 \%$ of activities recorded during the 24 -hour observations.

Finally, table 1 presents the comparative ratio between the relative number of errors and the frequency of activities for different categories of body systems and medical activities. In each of the categories presented in table 1 the percent of errors in this category, from the total number of errors, was divided by the percent of activities in this particular category from the total number of activities. A ratio of 1.0 implies that the relative frequency of errors in this category matched the relative frequency of activities. A ratio of $>1.0$ implies that the rate of errors of a particular type was larger than the respective frequency of activities. If the ratio is $<1.0$, the implication is that this particular category of activities was less susceptible to errors.

Examination of the results presented in table 1 indicates that input activities and central nervous system activities with error ratios of 2.38 and 1.76 , respectively, are the most error-prone categories. Cardiovascular system, urine, and digestive categories are on the low end of susceptibility to errors.

\section{DISCUSSION}

The ICU in the present study served as a model for examining the applicability of concepts and task analysis methods (adapted from human factors engineering) to the study of medical teams in clinical practice. Data collection techniques 
included the development of a general activity profile, accompanied by a detailed human factors analysis of the ICU workplace. These techniques served as anchors to calibrate and qualify the error reports recorded by the ICU medical staff. Human factors engineering, as a domain, focuses on the study of the interface between humans and their working environment, with a particular emphasis on technology. The main goal is to improve the match between technology, task requirements, and the ability of workers to cope with task demands. While the human factors engineering approach has been extensively employed in a wide variety of work environments, from the cockpit of an aircraft to the console of a nuclear power plant, the "health industry" has largely neglected this approach. Physicians and human factors experts may find it difficult to compare hospitals to factory assembly lines, which may be the reason that so little human factors engineering work has been done on daily hospital activity.

Only a handful of studies have been dedicated to human errors in critical care. Previous retrospective studies, ${ }^{4-8}$ using the hospital reporting system as a source for discovering errors, found only 92 instances of human errors (and 53 cases of equipment malfunction) in a period of 3 years. This finding represents an astonishingly low error rate, especially when compared with our results. However, such a difference can be expected when a prospective study with immediate reporting of errors by the medical staff is compared with a retrospective study of "filed" errors.

In our study, an average of 178 activities per patient were recorded daily, of which $1.7(0.95 \%)$ activities were judged erroneous. Assessing a $100 \%$ occupancy rate in six beds of the main ICU, at the period in which the study was conducted, it can be estimated that $>1000$ human errors were actually committed during the 4 months. During the same period, 476 human errors were self-reported by the medical and nursing staff. Or, close to $50 \%$ of the errors were discovered and self-reported. Considering the circumstances of the study, this rate of retrieval of human errors seems relatively high. Moreover, the findings of the three raters and the Technion staff were similar in terms of the errors and error category types for physicians and nurses. Such a similarity gives credence to the error reports in reducing the chances of biases or tendencies of selective reporting.

Twenty-nine percent (147) of the errors were graded as severe or potentially detrimental to the patients if not discovered in time (i.e. about two such errors in the ICU per 24 hours), which is an alarming finding. Such a finding underscores the crucial importance of the study of errors in the case of intensive medical care. At first glance-when considering the typical instability of ICU patients and the complex and demanding task of physicians and nurses-an overall error rate of $<0.5 \%$ (of which only $29 \%$ are rated severe) may be considered a high and reliable level of performance. However, when this error rate is calibrated by the large number of daily activities per patient, and the fact that the unit had only six beds, two severe errors per day cannot be taken lightly. In the rest of this discussion section, we attempt to qualify possible sources of errors.

Compared with nurses, physicians had a much higher rate of error. Recall that, of the total number of errors, $45 \%$ (238) were committed by physicians and 55\% (286) were committed by nurses, whereas physicians carried out only $4.7 \%$ (382) of the daily activities, while nurses carried out $84 \%$ (6857) of the activities. Three possible explanations for this finding-which may not be mutually exclusive-can be offered. One determinant may be the differences between physicians and nurses in the nature of their tasks. While nurses are mainly involved in routine and repetitive activities, physicians' activities comprise a much higher percentage of reactive and initiated interventions. These differences have been well documented in the unit activity profile. Initiated and reactive activities are, by default, less predictable and more susceptible to stress, and hence they also have increased probability for error.

Another contributing factor may be the fact that physicians in the present ICU, as part of their duties, were continually called on for emergency consultations outside the ICU. Consequently, their contact with each patient in the ICU was more intermittent. Also, each physician was expected to keep track of a larger number of patients in the ICU as compared with nurses. Each nurse was responsible for two patients, while physicians supervised all six beds. The greater the number of patients and the more intermittent the contact, the greater are the chances of confusion and error. Finally, due to the training role of the present ICU, as part of a university hospital, a considerable number of the physicians in the units were less experienced than the nurses.

Taken together, the above three factors highlight the critical importance of good communication and transfer of information between physicians and nurses. Since the responsibilities of physicians and nurses are complementary rather than overlapping, a complete, coherent, and updated knowledge base of the patient status requires a two-way information flow among team members. In addition, in the context of the studied ICU, it appears that nurses were involved in closer and more continuous monitoring of each patient than physicians. Thus, by serving as an active liaison, they can help physicians to bridge information gaps and avoid confusion. In the training environment, proper communication and exchange patterns may also enable residents and students to take advantage of the accumulated experience of nurses in unit conventions and routines.

The prevailing formal routes of written information exchange are the physician's order forms, daily follow-up sheets, and the patient's files. Physicians' rounds and nurses' shift changes are the formal occasions for verbal information transfer. Proper and complete exchange of written information entails keeping strict records of all activities on the relevant forms, while using adequately designed formats that are equally understandable to all involved team members. Regarding verbal briefings, it is highly desirable that nurses be included in the physicians' rounds and have a formal role in the information exchange. Shift changes should also be formalized in terms of the content and mode of information transfer.

Several outcomes of the present study indicated deviations from the above recommended conventions. The 24-hour observations showed that $18 \%$ of the activities were not recorded in any form. Verbal communications were only observed in $9 \%$ of the activities, while nurses and physicians conferred with each other only in $2 \%$ of the activities. Nurses were not an integral part of the physicians' rounds; in most cases, nurses did not participate, and were not included in the information exchange. These results should be considered, together with the diurnal distribution of errors (i.e. most errors occurred in morning hours, around the time of the physicians' morning rounds, with a 1-hour delay in the error peak of the nurses). One can only speculate at this time how this error peak could be influenced if nurses and physicians formally exchanged reports during morning rounds. Nurses had secondary error peaks at each shift change, which is another indication of problems in information exchange.

A related finding was that verbal exchanges between physicians and nurses were recorded in $37 \%$ of errors, which is an astonishing number, since verbal exchanges constituted $2 \%$ of the overall activity profile. Possibly, these communications reflect informal orders or information exchanges that were given under emergency circumstances. Such exchanges are more likely to be misunderstood and misperceived. Further systematic research is needed to examine this hypothesis. If this hypothesis is supported in future studies, it may strengthen the call for maintaining strict written records of all activities, and establish formal briefing procedures among staff members. 
The probability for error occurrence was not equally distributed among different types of activities. The data presented in table 1 indicate that input, output, and central nervous system-related activities were relatively more prone to the occurrence of errors. A contributing factor to the higher susceptibility to error of these categories may be the inadequate human factors design of the patient bedside as a work station, and deficiencies in the design of recording forms. Although a detailed discussion of this claim is beyond the scope of the present paper, such analysis has been conducted and reported elsewhere. ${ }^{9}$

The major problems demonstrated in this analysis were lack of standardization and congestion of instruments, monitors, wires, and intravenous catheters around the patient's bed. These problems often complicated access to the patient's bed and created problems of identification and status assessment. In addition, tubes, fluid bags, and drugs were insufficiently marked or had labels that were hard to read. Some of the recording forms were not specifically designed to answer the needs of the ICU unit, causing staff members to improvise and develop their own style of completing them. Other recording forms were deficient in terms of layout and clarity of display. It is easy to see why input and output activities-which mainly involve recording levels of drug and fluid administration, intravenous drip rates, and complex formulas on order forms-are especially susceptible to these types of communication problems. Therefore, it is expected that steps taken to alleviate these problems will lead to a reduction in the number of errors.

The higher error rates observed for central nervous systemrelated activities are believed to be influenced by other factors associated with the information-gathering and decisionmaking processes that are involved in performing central nervous system-related activities. A detailed discussion of these factors is presented in another paper. ${ }^{10}$

While our study offers important insights into the sources and nature of errors that occur in the ICU, along with practical steps for reducing the error rate, some cautionary words are in order. The mere conduct of a study of the kind reported in this article, with the accompanying observations and error reports, may change the behavior of staff members. Also, there are no conceivable control conditions, as it is impossible to "implant" errors and watch the time until their discovery. Finally, the study was conducted in a specific environmentthe ICU of an Israeli hospital, a unit grossly understaffed, which also serves as a training facility.

Although these features make this ICU somewhat different from ICUs in the United States and Europe, there is sufficient similarity, and the global research approach is still valid. To conduct a similar study, a unit must first become aware of the involved issues and the importance of studying errors. The unit may then proceed to seek the specific steps that need to be taken for conducting the study and applying the study's findings afterward.

In conclusion, the tools and concepts provided by cognitive psychology and human factors engineering proved to be both useful and powerful in the analysis of human errors in the demanding, dynamic, and complex environment of the ICU. We believe that the nature and causes of errors that were identified in our ICU were not unique and that they have general relevance for other ICUs, as well as for the analysis of medical units of other types.

\section{Authors' affiliations}

Y Donchin, M Biesky, C L Sprung, R Pizov, S Cotev, Department of Anesthesiology and Critical Care, Hadassah-Hebrew University Medical Center, Jerusalem, Israel

D Gopher, M Olin, Y Badihi, Department of Human Engineering, the Technion, Israel Institute of Technology, Jerusalem, Israel
This study was supported, in part, by a special grant from the Ministry of Labour and Social Affairs, The Committee for Research and Prevention in Occupational Safety and Health.

\section{REFERENCES}

1 Gaba DM: Human error in anesthetic mishaps. Int Anesthesiol Clin 1989;27:137-47.

2 Lenorovitz DR, Phillips MD: Human factors requirements engineering for air traffic control system. In: Salvendy G, ed. Handbook of human factors. New York: John Wiley, 1987, 1771-89

3 Brennan TA, Localio AR, Leape LL, et al. Identification of adverse events occurring during hospitalization. Ann Intern Med 1990;1 12:221-6.

4 Brennan TA, Leape LL, Laird NM, et al. Incidence of adverse events and negligence in hospitalized patients. N Engl J Med 1991;324:370-6.

5 Abramson NS, Wald KS, Grenvik ANA, et al. Adverse occurrences in intensive care units. JAMA 1980;244:1582-4.

6 Solomon SL, Wallace MB, Ford-Jones El, et al. Medication errors with inhalant epinephrine mimicking an epidemic of neonatal sepsis. N Engl Med 1984;310:166-70.

7 Knaus WA, Draper EA, Wagner DP, et al. An evaluation of outcome from intensive care in major medical centers. Ann Intern Med 1986;104:410-8.

8 Lesar TS, Briceland LL, Delcoure K, et al. Medication prescribing errors in a teaching hospital. JAMA 1990;263:2329-34

9 Gopher D, Olin M, Badihi Y, et al. The nature and causes of human errors in the medical intensive care unit. In: Proceedings of the Human Factor Society's 33rd Annual Meeting, 1989, 956-60.

10 Gopher D, Badihi Y, Donchin Y. Knowledge compilation by doctors and nurses on their patients and its relationship to errors. In: Proceedings of the 12th Triennial Congress of the International Ergonomics Association, Toronto, ON, Canada, 1994, 57-9.

\section{COMMENTARY}

\section{THE ROLE OF HUMAN FACTORS IN THE INTENSIVE CARE UNIT}

Once upon a time there were almost no formal investigations of the nature of human errors in hospitals. Today, however, there is an abundance of reports and a plethora of papers publishing robust data on this subject.

The paper by Donchin and colleagues ${ }^{1}$ was one of the first publications to investigate the nature of human errors in the intensive care unit (ICU), adopting approaches developed by human factors engineering. The ICU environment is complex, dynamic, with a constant change in time and stress. There is an excess of high technology equipment to facilitate diagnosis, monitoring and treatment of patients, but this often creates additional unexpected demands. The definition of human error in this study was vague, but this is the case in many classifications and taxonomies of human error-both with regard to genotype and phenotype. ${ }^{23}$ Even with more precise definitions it is difficult to achieve satisfactory descriptions and explanations of error in humans, given the complex nature of human interactions. Decision making by healthcare providers is influenced by factors such as workload, economy, ethics, and safety issues, which make the true picture much more difficult to fit neatly into a fixed taxonomy.

Looking through the newspapers from the last three decades, it is evident that human error is of crucial importance. It has been a critical factor in some of the most devastating events that have captured our attention, such as Chernobyl and Three Mile Island. ${ }^{4}$ Perhaps some of the most important lessons from these events were that they were the product of many different failures distributed widely in time. The errors or violations carried out by the operators on the night of 26 April 1988 in Chernobyl were just the last ingredients in the making of the disaster, actively breaching various barriers or safeguards. The contribution to the breakdown of a well defended but highly complex system can be divided into two categories: active and latent failures. ${ }^{2}$ Active failures are errors or violations by the persons who are directly working with the system, while latent failures are delayed actions or a lack of decisions related to design, organisation, or structure of the system. It was realised that nearly all accidents have roots 
in organisational and systemic root causes. These latent factors can be diagnosed and efforts towards avoiding repetition initiated. ${ }^{2}$

Fortunately, it has been realised that the efforts and work of multidisciplinary teams in the analysis of the nature of human error in the industrial sector can be transferred to the medical environment. This was prompted by increased attention to medical errors and, especially in the US, by rising malpractice insurance premiums. If this development should change health care, it is through reducing the events and their causes, and by developing methods to prevent errors or to attenuate their effect. $^{5}$

Donchin et $a l^{1}$ applied task analysis in an intensive care environment to describe the activities around the patients in a medical-surgical ICU, which was one of six critical care units in a tertiary care teaching hospital of 650 beds. The activities were observed and described by a team of non-medical investigators who had been specially trained for the purpose. Simultaneously, the physicians and the nurses were prompted to report any kind of errors occurring in the ICU. Error was defined as "any deviation from standard conduct, as well as addition or omission of action related to standard operating procedures or routines of the unit". The study demonstrated, on average, 178 activities per patient per day of which $0.95 \%$ were judged to be erroneous by the authors. The staff reported 554 human errors during the 4 month study period, $29 \%$ of which were graded as severe or potentially detrimental to the patient's well being if not corrected in time; $54 \%$ of the errors were committed by the physicians and $45 \%$ by the nurses. This was surprising as task analysis revealed that the physicians carried out only $4.7 \%$ of the activities. The activities carried out by the nurses tended to be more of a routine and repetitive character. The physicians were called away for emergency consultations and their contact with the patients was intermittent, hence they had an increased probability for error. In another subset of the task analysis the investigators found that verbal communication between nurses and physicians was the main mode of communication in only $2 \%$ of activities, but in $37 \%$ of these cases they recorded errors. The authors did not report the number of cases where crucial information should have been communicated but failed, but information transfer and degradation was clearly a problem.
Human factors engineering (HFE) is a discipline all healthcare personnel should know about in order to understand many aspects of patient safety. The concepts and tools of HFE can help an organisation to analyse adverse events and develop workable and effective countermeasures. HFE methods can also benefit healthcare personnel by moving them towards systems thinking and a culture of safety. ${ }^{6}$

The study by Donchin et al deserves special attention as it is one of the first and best examples of using a traditional engineering tool which has been known for many decades but only recently applied to health care. Task analysis is extremely useful in revealing the true nature of human errors in complex healthcare settings such as the ICU using trained observers. It shows how error rates are influenced by the diurnal distribution of activities, poor communication, and whether the activities are novel or routine. These simple lessons have many implications for the safety and quality of care delivered to our patients. Although we have known these lessons for 20 years, we have yet to implement many of them into the design of our hospitals, medical devices, healthcare work schedules, supervision of trainees, and communication between different healthcare providers.

P F Jensen

Department of Cardiothoracic Anaesthesia, The Heart Centre, Rigshospitalet, University of Copenhagen, DK-2100 Copenhagen, Denmark; foege@dadlnet.dk

P Barach

Department of Anesthesia and Critical Care, University of Chicago, Chicago, IL 60637, USA

\section{REFERENCES}

Donchin Y, Gopher D, Olin M, et al. A look into the nature and causes of human errors in the intensive care unit. Crit Care Med 1995;23:294-300.

2 Reason JT. Human error. New York: Cambridge University Press, 1990

3 Rasmussen J. The definition of human error and a taxonomy for technical system design. In: Rasmussen J, Duncan K, Leplat J, eds. New technology and human error. Chichester: John Wiley, 1987: 23-30.

4 Perrow C. Normal accidents. New York: Basic Books, 1984.

5 Brennan TA, Localio LL, Leape LL, et al. Incidence of adverse events and negligence in hospitalised patients. N Engl J Med 1991;324:370-6.

6 Gosbee JW, Lin L. The role of human factors engineering in medical device and medical system errors. In: Vincent $C$, ed. Clinical risk management. 2nd ed. London: BM Publications, 2000: 301-18. 